\title{
Discurso y práctica pedagógica en contextos de alto rendimiento escolar*
}

\author{
Discourse and Pedagogical Practice in High-performance School Frameworks \\ Discurso e prática pedagógica em contextos de alto rendimento escolar
}

\author{
Marco Antonio Villalta Páucar, ${ }^{a}$ Diego Palacios Díaz. ${ }^{b}$ \\ ${ }^{a}$ Escuela de Psicología, Facultad de Humanidades, Universidad de Santiago de Chile. \\ Fono: 56-2-27184370. Correo electrónico: marco.villalta@usach.cl \\ ${ }^{\text {b }}$ Escuela de Psicología, Facultad de Humanidades, Universidad de Santiago de Chile. \\ Fono: 56-2-27184370. Correo electrónico: diego.palacios@usach.cl
}

\begin{abstract}
RESUMEN
El objetivo del artículo es analizar la relación entre el discurso sobre la práctica pedagógica y la práctica real en sala de clases de los profesores en establecimientos educativos de alto desempeño y bajo nivel socioeconómico de Santiago de Chile. Se implementa un estudio descriptivo de metodología mixta, entrevistas semiestructuradas a nueve docentes, y análisis de filmación de 34 horas de clases. Se encuentra coherencia entre el discurso referido al contexto institucional y el tipo de intervención del profesor, y desfase entre el discurso sobre la interacción profesor-estudiante y el tipo de intervención con ellos en la clase.

Palabras clave: profesor, creencia, prácticas pedagógicas, relación profesor-alumno.
\end{abstract}

ABSTRACT
The aim of this paper is to analyze the relationship between the speech on teaching practice and the actual practice of teachers in educational institutions of high performance and low socioeconomic status in Santiago de Chile. A descriptive study of mixed methodology is implemented, with semistructured interviews of nine teachers, and film analysis of 34 hours of classes. There is coherence between the speech referred to the institutional context and the type of intervention by the teacher, yet there is a gap between the speech on teacher-student interaction and the type of intervention with their in class.

Key words: teacher, beliefs, teaching practices, teacher-student relationship.

\section{RESUMO}

Analisa-se a relação entre o discurso sobre a prática pedagógica e a prática real dos professores em sala de aula, em estabelecimentos educativos com alto desempenho e baixo nível socioeconômico, em Santiago do Chile. Desenvolve-se um estudo descritivo com metodologia mista, com entrevistas semiestruturadas com nove docentes e análise da filmagem de 34 horas das aulas dos mesmos. Encontra-se coerência entre o discurso do contexto institucional e o tipo de intervenção do professor e uma defasagem entre o discurso sobre a interação professoraluno e sobre o tipo de intervenção com os alunos em aula.

Palavras chave: professor, crença, práticas pedagógicas, relação professor-aluno.

* Estudio auspiciado por el Fondo Nacional de Ciencia y Tecnología (FONDECYT) del Gobierno de Chile, Proyecto $\mathrm{N}^{\circ} 1110478$. 


\section{INTRODUCCIÓN}

Se encuentra un desfase entre lo que los profesores dicen y lo que efectivamente hacen como práctica pedagógica en sala de clases (Torrado y Pozo, 2006). Esta diferencia entre discurso y acción no es propia del campo educativo, es una realidad inherente a los estudios sociales, dado que el decir y el hacer respecto a algo está determinado por sus propias condiciones de realización (Taylor y Bogdan, 1998). La práctica cotidiana está influenciada por contingencias del contexto socio-espacial que no siempre pueden ser recogidas en el discurso que comúnmente se mueve en el campo de las explicaciones y las intenciones.

El séptimo Informe sobre Desarrollo Humano en Chile (Programa de las Naciones Unidas para el Desarrollo [PNUD], 2009) analiza las prácticas cotidianas que obstaculizan y aquellas que potencian el desarrollo humano, definiendo las prácticas como "modos de actuar y relacionarse que despliegan las personas en espacios concretos de acción" (PNUD, 2009: 15), que articulan reglas institucionales, la subjetividad y el conocimiento práctico; el "saber hacer". Estos tres elementos son las fuerzas que definen las posibilidades de desarrollo de las prácticas. Es el enfoque para el análisis de los discursos sobre la práctica que se considera en el presente estudio.

En la educación, la reglas institucionales son constitutivas de la cultura institucional escolar, las cuales enmarcan los comportamientos, promueven valores, generan rituales y preservan tradiciones (Baeza, 2008). La cultura institucional es síntesis histórica de la acción coordinada de los actores educativos que se encuentran en el espacio escolar (Pérez Gómez, 1998). La subjetividad refiere al sentido personal que el sujeto da a su práctica, la cual es construida en la interacción con otros (PNUD, 2009). En tal sentido, el profesor construye su rol y tarea en el aprendizaje de los estudiantes, desde la experiencia personal relacionada al contexto en cual trabaja (Villalta, Martinic y Guzmán, 2011), y a sus teorías implícitas sobre el aprendizaje, adquiridas en la vida cotidiana de la escuela (Pecharromán y Pozo, 2006).

El conocimiento práctico, fuertemente enmarcado por la cultura, reduce la incertidumbre y delimita las expectativas (PNUD, 2009); es el "saber hacer"-aunque no siempre eficaz- significativo para los actores educativos y, en consecuencia, resistente al cambio. Desde esta perspectiva se puede entender, por ejemplo, que las prácticas frontales de enseñanza sean hegemónicas en las aulas chilenas a pesar de que se modificaron las directrices nacionales de enseñanza e introdujeron nuevos recursos pedagógicos en el aula (Martinic y Vergara, 2007).

El discurso del profesor sobre su práctica pedagógica integra una forma particular de interpretar la cultura institucional escolar, con sus creencias sobre identidad y rol en el proceso de enseñanza y aprendizaje, y con el oficio cotidiano de la enseñanza; lo cual confluye en una teoría sobre el "saber hacer" que, aunque posiblemente no tiene relación directa con la práctica pedagógica real en el aula, sí define las posibilidades de desarrollo de las mismas (Torrado y Pozo, 2006).

El objetivo del artículo es analizar el discurso sobre la práctica pedagógica en relación a la práctica real en sala de clases que tienen los profesores de educación media en establecimientos educativos de alto desempeño y de bajo nivel socioeconómico de la Región Metropolitana. Es una investigación de tipo cualitativo, que toma como fuente de información 9 entrevistas semiestructuradas a profesores respecto a sus prácticas pedagógicas de aula en establecimientos educativos de alto desempeño, así como la filmación de las mismas. Son dos las preguntas que orientan el estudio: ¿qué elementos 
del discurso constituyen la práctica pedagógica del docente en contextos educativos de alto rendimiento escolar?, y ¿cómo estos elementos discursivos se ponen de manifiesto en la práctica pedagógica en el aula?

En el segundo título se exponen los referentes teóricos sobre cultura escolar y creencias del profesor sobre las prácticas y aprendizaje escolar; en el tercer título se presenta la metodología de estudio. A continuación, en el cuarto título se presentan los resultados del análisis de los discursos y del análisis de filmación de las clases observadas. Finalmente, en el quinto título se presenta la discusión y conclusiones del estudio.

\section{REFERENTES TEÓRICOS}

\subsection{CULTURA ESCOLAR Y APRENDIZAJE}

Según lo señala Baeza, la cultura escolar "es un sistema subyacente de normas, valores, rituales, tradiciones, ceremonias e historias que se acumulan a través del tiempo" (2008: 198), compartido por profesores, estudiantes y apoderados, y que configura determinadas expectativas, creencias y acciones. Es una construcción, síntesis histórica del "cruce de culturas" (Pérez Gómez, 1998) de quienes participan de ella e interactúan en el espacio y tiempo de la escuela.

Desde esta perspectiva, existe una estrecha relación entre la cultura, en tanto ethos que impregna la vida en la escuela, y las prácticas sociales para convivir, enseñar y aprender en el espacio escolar. En este ethos coexisten diversas subculturas, como por ejemplo la cultura del docente, que define modos específicos de las prácticas educativas (Hargreaves, 2005), las que se encuentran asociadas a los aspectos estructurales de la organización de la escuela, y aportan a comprender el logro de los resultados educativos.

La relación entre cultura y aprendizaje escolar ha sido documentada en diversos estudios para explicar el fracaso o el éxito educativo, destacando aspectos como el ambiente socioafectivo a nivel institucional, los niveles de autonomía que permite la estructura organizacional del establecimiento, y las posibilidades de profesionalizar el trabajo docente (Cornejo y Redondo, 2007); también los juicios positivos compartidos sobre la identidad institucional, tales como metas centradas en el aprendizaje, claridad en las normas de comportamiento al interior del establecimiento y las condiciones de infraestructura educativa (Villalta, 2014).

La segmentación socioeconómica de la educación en Latinoamérica, especialmente en Chile, conlleva notorias diferencias en la calidad de la formación que reciben los estudiantes, diferencias de capital cultural y, en consecuencia, la generación de expectativas y resultados de aprendizaje en relación directa con la inequidad estructural y simbólica del país (Baeza, 2008). La pobreza socioeconómica y de capital cultural -aspectos que configuran realidades sociales vulnerables a la exclusión social- está asociado a fracaso escolar (Ruíz y García, 2007).

Por una parte, en los establecimientos que operan en sectores sociales vulnerables, el fracaso escolar suele ser atribuido además a las insuficientes capacidades cognitivas de los estudiantes, a su poca persistencia para enfrentar y superar dificultades, la baja autoestima, la carencia de redes sociales y de socialización primaria que les otorgue los códigos culturales para participar de modo efectivo en la escuela (Román, 2003). 
Por otra parte, se encuentra que los cambios intencionados para el logro de aprendizaje en establecimientos educativos en contextos sociales vulnerables, en aspectos de la cultura escolar tales como el mejoramiento de los procesos organizacionales y las actividades de sala de clases, están asociados a logros de aprendizaje escolar (Volante et al., 2008). Esto pone en evidencia, por un lado, que la cultura escolar está constituida de patrones organizacionales aprendidos y, por lo tanto, éstos puede cambiar (Pirela de Faría y Sánchez de Gallardo, 2009); y, por otro lado, que el fracaso escolar en estos contextos es un tema de inequidad social y de calidad educativa, no de capacidades de los sujetos para aprender.

Los estudios sobre la relación virtuosa entre cultura y aprendizaje escolar destacan aspectos como la relación emocional positiva entre profesores y estudiantes (Laboratorio Latinoamericano de Evaluación de la Calidad de la Educación [LLECE], 2010); el análisis colectivo para la toma de decisiones institucionales que contribuyen a una identidad institucional positiva (UNICEF y Ministerio de Educación de Chile [MINEDUC], 2004); prácticas de enseñanza sustentadas en el análisis de la experiencia profesional y que valoran la diversidad de los estudiantes (Murillo y Román, 2009); la satisfacción docente, elemento asociado a la promoción de climas positivos en el aula (Fernández y Cuadrado, 2008), entre otros.

La relación entre cultura y aprendizaje escolar tiene como punto de encuentro vital los sucesos de sala de clases, lo cual ha sido foco de intervención para el mejoramiento educativo (Volante et al., 2008), espacio-tiempo donde se constituye la práctica pedagógica del docente (Villalta, Martinic y Guzmán, 2011).

\subsection{PENSAMIENTO DEL PROFESOR Y PRÁCTICAS DE ENSEÑANZA}

Las concepciones de los docentes acerca del aprendizaje y la enseñanza son elementos de central importancia al momento de comprender las conductas y prácticas que los profesores desarrollan en las aulas de clases. Diversos enfoques de investigación han estudiado cuáles son los procesos cognitivos que orientan las acciones del docente; diversidad de enfoques que han sido agrupados en lo que se ha denominado el paradigma del pensamiento del profesor (Marcelo, 1989). De acuerdo a Medina (2006), es posible reconocer dos tendencias en el estudio del paradigma del pensamiento del profesor: a) de los contenidos -diversos constructos que configuran el pensamiento educativo y la dimensión personal del profesorado: creencias, teorías implícitas, representaciones sociales, entre otros-, y b) de los procesos -es decir, la forma en que dichos constructos son modificados por las experiencias cotidianas del docente-.

El tema del pensamiento del profesor se ha estudiado predominantemente desde la perspectiva de las creencias y las teorías implícitas que los docentes tienen acerca de distintos aspectos del proceso de enseñanza y aprendizaje. Se ha considerado que dichas creencias configuran teorías implícitas, esto es, un sistema o perspectiva conceptual personal e idiosincrático que permite a los docentes afrontar las diversas situaciones que se presentan en el contexto escolar (Medina, 2006), a partir de las cuales se ha buscado comprender por qué los docentes actúan de determinada manera en el ambiente de la sala de clases. Al respecto, se encuentra que las creencias tienen afinidad con la cultura social en tanto guía de la conducta de las personas (Chan \& Elliott, 2002), y es posible encontrar disonancia entre lo que la persona cree y lo que efectivamente hace (Torrado y Pozo, 2006). 
En relación al aprendizaje escolar, se encuentra que las teorías implícitas que tienen los profesores acerca del aprendizaje ponen de manifiesto, entre otros aspectos, fuerte presencia de realismo ingenuo sobre el conocimiento, es decir, la idea del conocimiento como copia de la realidad (Martin et al., 2006); que las prácticas de enseñanza tienen distinto nivel de consistencia con la visión de mundo que manifiestan los profesores, y que se adaptan a las creencias sobre los dominios generales y específicos que es necesario desarrollar en el aprendizaje (Olafson \& Schraw, 2006); que hay diferencias entre profesores con experiencia y estudiantes de pedagogía respecto al papel de los enfoques constructivistas en el proceso de enseñanza (Gómez y Guerra, 2012); que las creencias sobre el aprendizaje son diferenciadas desde la formación inicial para el profesor según el nivel de especialización -Educación Básica o Educación Media- (García y Sebastián, 2011); que las creencias cambian según el significado personal que tiene para el profesor su experiencia laboral en contextos sociales vulnerables (Barría et al., 2010), y; que las creencias tienen relación con procesos específicos de aprendizaje (Makuc, 2008).

En síntesis, los estudios ponen de relieve, en primer lugar, que generalmente lo que los docentes piensan o creen no es siempre lo que realmente realizan en sus prácticas pedagógicas; aunque, no obstante ello, se debe reconocer y valorar que las creencias y teorías implícitas juegan un importante rol en términos de su función orientadora de la conducta de las personas. En segundo lugar, se pone en evidencia el valor altamente idiosincrático y contextual de las teorías implícitas, puesto que la evidencia empírica muestra de forma clara cómo estas concepciones varían y se adaptan según las particularidades contextuales y las demandas específicas a las que los docentes se ven enfrentados en su experiencia profesional cotidiana (Leal et al., 2009).

Conocer las prácticas de enseñanza desde el discurso y la práctica real del profesor es una forma de abordar las disonancias encontradas entre discurso y práctica pedagógica partiendo de los aspectos donde ambas confluyen: se trata de acciones racionales que constituyen el ethos cultural de la institución escolar puesta de manifiesto en la sala de clases. Esto abre el desafío de desarrollar un abordaje metodológico que sitúe los procesos cognitivos del profesorado en una perspectiva ecológica para explicar las prácticas e interacciones que se dan en el contexto escolar, y que ofrece pautas para el comportamiento de los actores educativos. La comprensión de lo que los profesores creen y piensan sobre los fenómenos educativos, es un paso necesario para comprender cómo actúan en las aulas de clases.

\section{MÉTODO}

Es un estudio de enfoque descriptivo interpretativo metodológicamente mixto, que combina técnicas de análisis cualitativas y herramientas cuantitativas en función de las preguntas y objetivo del estudio.

\subsection{PARTICIPANTES}

Los casos de estudio se describen a dos niveles interdependientes, el contexto o escenario de las prácticas pedagógicas -los liceos-, y los casos de estudio propiamente tales -informantes-. El escenario de estudio está compuesto por liceos con las siguientes características: 
- Liceos de Alto Desempeño Académico. Se toma como criterio de inclusión aquellos que poseen subvención por Desempeño de Excelencia otorgado por el Sistema Nacional de Evaluación de Desempeño de Chile, cuyos criterios definen contextos favorables para lograr altos resultados de aprendizaje (MINEDUC, 2010).

- Nivel Educativo. 2do Medio, de curriculum Científico Humanista y Técnico Profesional, pues es el nivel educativo de quienes rinden la prueba de evaluación del Sistema de Medición de Calidad de la Educación (SIMCE). Esto permite la comparación de logro educativo entre los establecimientos y estudiantes chilenos.

- Grupo Socioeconómico Medio Bajo. Determinado según la base de datos de la prueba nacional SIMCE 2010 en 2do Medio de acceso público en internet (http://www.simce.cl/).

- Puntaje SIMCE 2010. Específicamente en 2do medio, por encima del Promedio Nacional y de sus pares institucionales y sociales en Matemáticas y Lenguaje. Eso asegura tomar establecimientos con altos logros de aprendizaje escolar (http://www.simce.cl/).

Los casos de estudio fueron profesores seleccionados con los siguientes criterios:

a) Eficacia. Los profesores son nominados por sus pares o por el Equipo Directivo del establecimiento como "eficaces" en su labor profesional, en la generación de clima laboral positivo, en la relación con sus estudiantes y en el logro de resultados de aprendizajes.

b) Experiencia. Con cinco años o más de experiencia docente. Preferentemente en el mismo establecimiento para asegurar la relación cultura y práctica pedagógica en la explicación de la efectividad escolar.

c) Voluntad. Para participar del estudio se requiere que el/la profesor/a permita el acceso a la filmación de sus clases.

Se trabajó con 9 profesores de diversas disciplinas y se filmaron 3 clases consecutivas de cada uno de ellos (Tabla 1):

Tabla 1. Liceo, Modalidad Curricular, Nivel Socioeconómico, $\mathrm{N}^{\circ}$ de profesores, $\mathrm{N}^{\circ}$ de clases filmadas y resultados SIMCE 2010 de establecimientos participantes

\begin{tabular}{|c|l|l|c|c|c|c|c|}
\hline $\mathbf{N}^{\circ}$ & Liceo $^{1}$ & Modalidad & $\begin{array}{c}\text { Nivel } \\
\text { socioeconómico }\end{array}$ & $\begin{array}{c}\text { Profesores } \\
\text { participantes }\end{array}$ & $\begin{array}{c}\mathbf{N}^{\circ} \text { total de } \\
\text { clases filmadas }\end{array}$ & \multicolumn{2}{|c|}{ SIMCE 2010 } \\
\cline { 6 - 8 } 1 & SSB & $\begin{array}{l}\text { Técnico } \\
\text { Profesional }\end{array}$ & Medio bajo & 2 & 6 & 264 & 278 \\
\hline 2 & EMP & $\begin{array}{l}\text { Científico } \\
\text { Humanista }\end{array}$ & Medio bajo & 2 & 6 & 278 & 285 \\
\hline 3 & CJDC & $\begin{array}{l}\text { Científico } \\
\text { Humanista }\end{array}$ & Medio & 3 & 9 & 287 & 296 \\
\hline 4 & SJPS & $\begin{array}{l}\text { Técnico } \\
\text { Profesional }\end{array}$ & Medio Bajo & 2 & 6 & 293 & 303 \\
\hline
\end{tabular}

Fuente: elaboración propia.

En las identidades de los establecimientos se coloca un seudónimo para preservar el compromiso ético de proteger la confidencialidad de los informantes. 


\subsection{TÉCNICAS DE RECOLECCIÓN DE INFORMACIÓN}

Fueron consideradas para el estudio las siguientes técnicas de recolección de datos:

- Entrevistas en profundidad con docentes. Se utilizaron a fin de conocer sus representaciones de la práctica pedagógica, los procesos cognitivos y conocimiento escolar que definen los objetivos pedagógicos con los cuales planifican su quehacer en el aula. Permitieron también conocer, desde la perspectiva de los docentes, las competencias profesionales vinculadas a sus prácticas pedagógicas. Se realizaron 9 entrevistas en profundidad.

- Observación/filmación de sala de clases. Para analizar el tipo de intervención de docentes y estudiantes en el aula se observaron 3 sesiones por docente, las cuales fueron previamente acordadas con el/la profesor/a. Para el presente estudio se analizan en total 27 sesiones de registro de observación de las interacciones en la sala de clases.

\subsection{PROCEDIMIENTO DE ANÁLISIS DE DATOS}

Las entrevistas se analizaron mediante los principios de codificación Abierta y Axial de la Teoría Fundamentada (Strauss y Corbin, 2002) y el análisis Semántico de Contenido en perspectiva estructural (Navarro y Díaz, 1999): se realiza codificación abierta de los discursos, los cuales, en la codificación axial, son puestos en relación intra-textual con unidades de contexto extra-textual que estudios previos indican como estructurantes de la práctica cotidiana (PNUD, 2009; Villalta y Martinic, 2011): la subjetividad, el conocimiento práctico y la cultura institucional. La codificación axial permite establecer hipótesis teóricas sobre la relación entre las codificaciones abiertas y de éstas con los contextos de referencia, las cuales en su conjunto estructuran la práctica pedagógica.

Las filmaciones fueron analizadas según la intervención de profesor/a y estudiantes. La intervención es una categoría del Análisis de la Conversación (Villalta, 2009) para describir el proceso comunicativo de la clase. En la perspectiva conversacional, la intervención es la participación de cada interlocutor en relación de interdependencia ilocutoria para la construcción del proceso de enseñanza y aprendizaje. Para el análisis de las categorías de intervención de profesor (Tabla 2) y estudiantes (Tabla 3) se recogen los aportes de estudios previos (Villalta, Assael y Martinic, 2013). El análisis se hizo con apoyo computacional del software Videograph (Rimmele, 2009).

Tabla 2. Intervenciones del profesor vinculadas al proceso cognitivo y construcción del conocimiento escolar

\begin{tabular}{|l|l|}
\hline Intervención del profesor & \multicolumn{1}{c|}{ Definición } \\
\hline $\begin{array}{l}\text { Presenta conocimientos } \\
\text { escolares }\end{array}$ & $\begin{array}{l}\text { Explicita objetivos, reformula su propia intervención, uso de gestos } \\
\text { para captar atención del estudiante y enfatizar aspectos de los } \\
\text { contenidos (Cuadrado y Fernández, 2008), grafica contenidos en la } \\
\text { pizarra o con uso de sistemas computacionales. }\end{array}$ \\
\hline $\begin{array}{l}\text { Favorece participación } \\
\text { del estudiante }\end{array}$ & $\begin{array}{l}\text { Expresiones afirmativas, repeticiones de la intervención del estudiante, } \\
\text { gestos de apoyo como asentimiento con la cabeza, pasear por la sala } \\
\text { atento a posibles consultas (Cuadrado y Fernández, 2008). }\end{array}$ \\
\hline
\end{tabular}




\begin{tabular}{|l|l|}
\hline Reelaboración & $\begin{array}{l}\text { Retoma la intervención de uno o más estudiantes para mostrar una } \\
\text { visión más amplia del concepto que se trabaja (Cuadrado y Fernández, } \\
\text { 2008). }\end{array}$ \\
\hline Síntesis & $\begin{array}{l}\text { Resumen de cuestiones más relevantes del contenido que se trabaja } \\
\text { (Cuadrado y Fernández, 2008), conecta conceptos presentados. }\end{array}$ \\
\hline $\begin{array}{l}\text { Pregunta de dominio } \\
\text { de contenido }\end{array}$ & $\begin{array}{l}\text { Pide al estudiante que elija una respuesta entre un conjunto de } \\
\text { alternativas, o que responda con el uso de información factual o } \\
\text { conceptual (Nathan \& Kim, 2009). }\end{array}$ \\
\hline $\begin{array}{l}\text { Preguntas de elaboración } \\
\text { de contenido }\end{array}$ & $\begin{array}{l}\text { Pide al estudiante que elabore una respuesta que conecte o aplique lo } \\
\text { aprendido a nuevas situaciones (Nathan \& Kim, 2009). }\end{array}$ \\
\hline Preguntas de metaproceso & $\begin{array}{l}\text { Pide al estudiante que justifique, explique, que desarrolle su } \\
\text { intervención, o una intervención previa (Nathan \& Kim, 2009). }\end{array}$ \\
\hline Evaluación cerrada & $\begin{array}{l}\text { Evaluación breve de aceptación o rechazo de respuesta del estudiante } \\
\text { (Wells \& Mejía, 2005). }\end{array}$ \\
\hline Evaluación elaborada & $\begin{array}{l}\text { El profesor confirma la respuesta (adecuada o no) del estudiante e } \\
\text { incorpora nuevas elaboraciones a dicha respuesta (Wells \& Mejía, } \\
\text { 2005), o pregunta solicitando más información }\end{array}$ \\
\hline
\end{tabular}

Fuente: elaboración propia.

Tabla 3. Intervenciones del profesor vinculadas al proceso cognitivo y construcción del conocimiento escolar

\begin{tabular}{|l|l|}
\hline Intervención del estudiante & \multicolumn{1}{c|}{ Definición } \\
\hline $\begin{array}{l}\text { Validación de conocimientos } \\
\text { escolares }\end{array}$ & $\begin{array}{l}\text { Expresiones verbales ("ya", "aja", repetición textual de lo que dice } \\
\text { el profesor, o completamiento de frases, etc.) y no verbales (tomar } \\
\text { nota, mirar al profesor, afirmar con la cabeza, etc.) que validan el } \\
\text { contenido que entrega el profesor (Cobo, 1998). }\end{array}$ \\
\hline Reelaboración & $\begin{array}{l}\text { Retoma la intervención de uno o más estudiantes, o del profesor, } \\
\text { para dar su visión del concepto que se trabaja. }\end{array}$ \\
\hline Propone & $\begin{array}{l}\text { Aporta nuevos elementos (habla de su experiencia, relaciona con } \\
\text { aprendizajes previos) a la clase. }\end{array}$ \\
\hline $\begin{array}{l}\text { Responde a dominio } \\
\text { de contenido }\end{array}$ & $\begin{array}{l}\text { Elige una de las alternativas que presenta el profesor, produce una } \\
\text { respuesta en los términos de conocimiento factual o conceptual } \\
\text { que solicita el profesor. }\end{array}$ \\
\hline $\begin{array}{l}\text { Responde con } \\
\text { elaboración de contenido }\end{array}$ & $\begin{array}{l}\text { Elabora una respuesta para resolver una situación didáctica (la } \\
\text { respuesta a preguntas abiertas, las respuestas a ejercicios de una } \\
\text { guía, la interpretación de un texto, etc.). }\end{array}$ \\
\hline Metaproceso & $\begin{array}{l}\text { Habla sobre su propio razonamiento, vincula su intervención con } \\
\text { intervenciones previas. }\end{array}$ \\
\hline Relación social & $\begin{array}{l}\text { Intervenciones que, en principio, no tienen ninguna relación con } \\
\text { los contenidos escolares trabajados en clases. }\end{array}$ \\
\hline En colectivo & $\begin{array}{l}\text { No hay diálogo directo reconocible entre profesor y algún } \\
\text { estudiante. }\end{array}$ \\
\hline
\end{tabular}

Fuente: elaboración propia. 


\section{RESULTADOS}

Los resultados se presentan en dos dimensiones: 1) el análisis de contenido de las entrevistas, y 2) el análisis de las intervenciones en el aula. Ambas dimensiones se ponen en relación en el punto Conclusiones.

\subsection{ANÁLISIS DE CONTENIDO DE ENTREVISTAS}

Las categorías abiertas se organizan en tres campos discursivos, los cuales se encuentran interrelacionados y estructuran la práctica pedagógica. El primero de ellos refiere al campo discursivo de la subjetividad, que agrupa un conjunto de textos que refieren a experiencias personales de los profesores, que se subdividen en dos categorías, una denominada formación continua, textos que indican el valor que los docentes dan a los cursos de postgrados o perfeccionamientos para sus prácticas profesionales de aula:

"[...] a través de mis veinticinco años, yo creo que veintiuno o veintidós años siempre he estado estudiando algo, siempre he estado en algún curso de perfeccionamiento" (Profesora, JDC).

La segunda categoría del campo discursivo subjetividad es denominada modelo docente, que contiene textos que refieren a la síntesis que hace el profesor de su experiencia como alumno, desde donde extrae una forma positiva de valorar la práctica en tanto intervención para abrir perspectivas de mundo y desafiar al estudiante:

“... Tuve profesores $[\ldots]$ que me decían, "mira, esto es así, pero si tú lo tomas de este otro punto de vista puede que no sea asî", o sea, ahí yo fui aprendiendo ciertas cosas, y esto, yo te estoy hablando de la época del colegio, que después se potenciaron mucho más en la Universidad" (Profesor, REMP).

El segundo campo discursivo es el denominado del conocimiento práctico, que en el caso de los profesores se relaciona con dos categorías: (a) conocimiento de la disciplina que enseña, y (b) conocimiento de la interacción con los alumnos que le permite liderar el proceso comunicativo en la sala de clases.

La categoría (a) está descrita por la sub-categoría especificidad del saber que refiere el valor que el docente otorga a los saberes de la disciplina que enseña, diferenciándolo de otros saberes escolares. A continuación se presentan dos ejemplos de las disciplinas de Lenguaje e Historia:

"De partida el lenguaje se usa en todas las asignaturas, un alumno, o una persona que no maneje las herramientas mínimas o las competencias mínimas del lenguaje le va a costar mucho entender historia, le va a costar entender ciencias [...]" (Profesor Lenguaje, REMP).

“... lo característico de la historia es que hay contenidos que tú puedes explicar en el presente [...], el contenido histórico yo lo pongo en que los cabros desarrollen habilidades, por ejemplo un análisis comparativo, por ejemplo una síntesis, por ejemplo una evaluación, una interpretación..." (Profesor Historia, JDC). 
El conocimiento de la disciplina también está descrito por la sub-categoría estrategia de enseñanza, la cual, no obstante la especificidad disciplinar, comparte características de sistemático y desafiante para los estudiantes,

"[...] yo presento mi clase sistematizada y ordenada [...] entonces yo siempre presento una propiedad de algo, yo después lo demuestro, lo ejemplifico y digo: "ya, ahora te toca a ti"" (Profesor, JDC).

La categoría (b) conocimiento de la interacción con los alumnos permite a los profesores discernir acciones que describen la sub-categoría promover la participación en el aula, la cual es una invitación abierta y constante a todos:

“... debería haber alguna pregunta, debería haber algún... que pase una hora y media y no me pregunten nada... o sea no es una buena clase, no para mí [...], entonces uno también tiene que tratar de soltar un poquito eso, preguntarle al niño que nunca te preguntó, hablar con la persona que no participa" (Profesor, SJ).

Asimismo, la categoría (b) se estructura con la definición de determinadas prácticas que caracterizan al docente, las cuales se agrupan dentro de la sub-categoría cualidades del profesor, y refiere a los siguientes elementos: 1) establecer relación afectiva con los estudiantes, 2) comunicación efectiva, y 3) verificación constante de los aprendizajes:

"Trato de establecer una relación más dialógica con los alumnos en la medida de lo posible" (Profesor, REMP).

"Si el profesor se ve dubitativo los cabros no enganchan, no hay una empatía" (Profesor, JDC).

"[...] Todavía me emociono cuando un alumno me dice "Me acuerdo cuando usted me dijo" [...] asocian cosas de antes, en clases posteriores lo sacan otra vez, entonces igual, lo tienen, lo tienen, lo incorporaron". (Profesora, SSB).

El tercer campo discursivo es el contextual, que refiere al ámbito de la cultura escolar institucional que contiene y sostiene las prácticas pedagógicas de aula. Dos sub-categorías referidas al aprendizaje escolar constituyen la descripción del contexto: a) la disciplina, entendida como el orden y cumplimiento de normativas:

“(...) mantener ese orden, esta disciplina, esa constancia [...] el respeto en la clase, sobre todas las cosas, y también es importante manejarse con los chiquillos y darles confianza, decirles que no existen obstáculos" (Profesor, JDC).

Y b) la sistematicidad del proceso de enseñanza, que refiere a las acciones de planificación y evaluación constante de las acciones y resultados en el aula:

“[...] La planificación de nuestras clases está hecha de manera centralizada, [...] y ésa es como mi hoja de ruta para organizar mis tiempos, organizar mis materiales y todos los recursos que utilizo en clases" (Profesor, REMP). 
Las categorías y subcategorías discursivas descritas son componentes de la práctica pedagógica que tiene como punto de mayor confluencia el conocimiento que el profesor tiene de la interacción con los estudiantes en el aula. Considerando el enfoque de estudio de las prácticas que propone el PNUD (2009), dichas categorías son organizadas en campos discursivos que refieren a la experiencia personal extra-aula -campo discursivo de subjetividad-, al conocimiento práctico de los sucesos de sala de clases -campo del conocimiento práctico-, y al contexto institucional extra-aula -campo discursivo contextual-, cuyas categorías operan como un todo sistémico (Figura 1).

Figura 1. Elementos del discurso docente que estructuran la práctica pedagógica

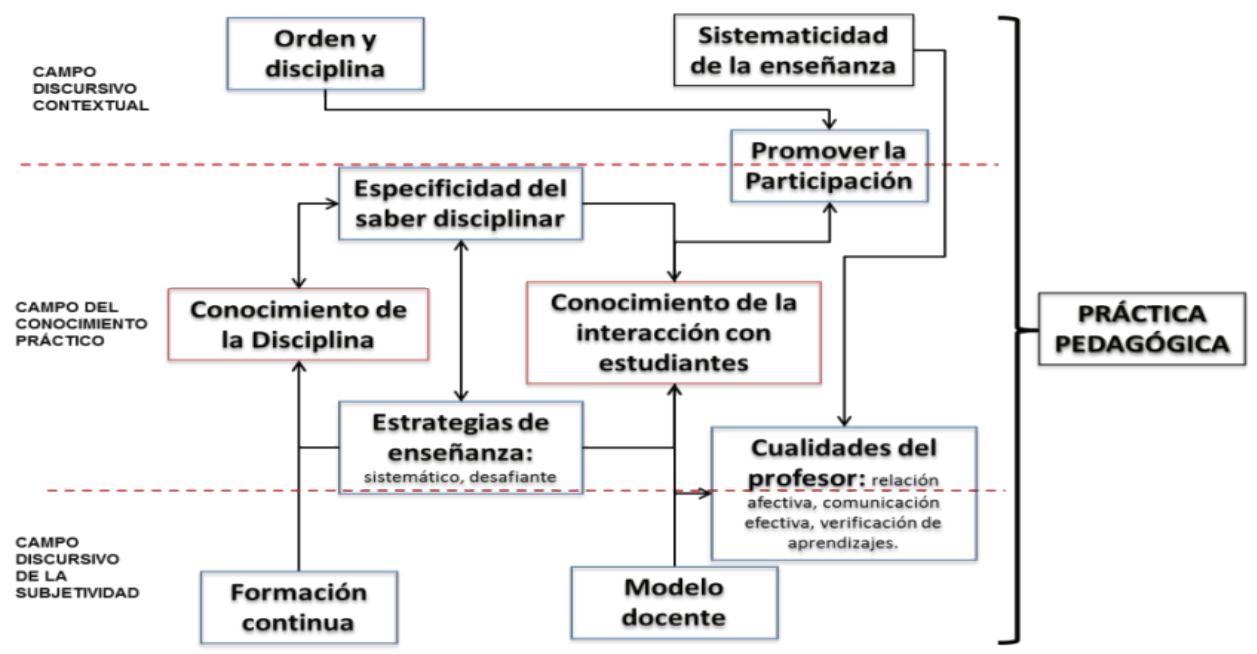

Fuente: elaboración propia.

La puesta en relación de las categorías y sub-categorías definidas permite comprender que la formación continua que describen los profesores fortalece el conocimiento de la disciplina escolar que enseñan, permitiéndoles delimitar el valor y la especificidad de dicho saber, así como también las estrategias de la enseñanza.

El conocimiento de la disciplina escolar no es suficiente para constituir el "saber hacer" de la práctica pedagógica en el aula. Se requiere del conocimiento de las interacciones con los estudiantes, esto es, la forma eficaz de relacionarse con ellos, para lo cual el profesor apela a su experiencia personal y profesional de donde construye su modelo docente, el cual le da sustentos prácticos para dos acciones: (a) promover la participación de los estudiantes en el aula, y (b) para discernir las cualidades del profesor en dicho contexto. No obstante, dichas acciones se sostienen o validan en dos elementos que caracterizan la cultura escolar de los establecimientos de alto rendimiento educativo: (1) el orden y la disciplina, lo cual enmarca los comportamientos posibles y deseables para ser miembro integrante de la comunidad escolar, y (2) la sistematicidad de la enseñanza, la cual se organiza y tiene soportes a nivel institucional. 


\subsection{ANÁLISIS DE LAS FILMACIONES DE CLASES}

Se filmaron tres sesiones consecutivas por cada docente, acumulándose un total de 27 clases. Cada clase duró en promedio una hora con 18 minutos. Las intervenciones del profesor y los estudiantes fueron categorizadas cada cinco segundos en todas las clases. Se observó en total 34 horas y 24 minutos de filmación.

Tabla 4. Frecuencia porcentual de tiempo de intervenciones del profesor en las clases

\begin{tabular}{|l|c|c|}
\hline \multicolumn{1}{|c|}{ Categorías de Intervenciones del Profesor } & $\begin{array}{c}\text { Frecuencia } \\
\text { (unidades de 5 segundos) }\end{array}$ & Porcentaje \\
\hline 1. Presenta conocimientos escolares. & 13.461 & 54,3 \\
\hline 2. Favorece participación de estudiantes. & 8.025 & 32,4 \\
\hline 3. Reelabora intervención de estudiantes. & 184 & 0,7 \\
\hline 4. Síntesis de los contenidos que presenta en clases. & 20 & 0,1 \\
\hline 5. Pregunta de dominio de contenidos factuales. & 599 & 2,4 \\
\hline 6. Pregunta que requiere elaboración de contenidos. & 330 & 1,3 \\
\hline 7. Preguntas de metaproceso. & 123 & 0,5 \\
\hline 8. Evaluación cerrada, breve de respuesta. & 73 & 0,3 \\
\hline 9. Evaluación elaborada, profundiza en la respuesta. & 106 & 0,4 \\
\hline Total (unidades válidas) & $\mathbf{2 2 . 9 2 1}$ & $\mathbf{9 2 , 4}$ \\
\hline
\end{tabular}

Fuente: elaboración propia.

En el 92\% del tiempo total de clases observadas fue posible categorizar las intervenciones de los profesores en las clases (Tabla 4). El análisis de las intervenciones de los profesores indica que el 54,3\% del tiempo de las clases los profesores presentaron conocimientos escolares (categoría 1), y el 32,4\% del tiempo intervino para favorecer la participación del estudiante en la clase (categoría 2), con el empleo de gestos afirmativos a la intervención del estudiante, recorrer la sala para atender preguntas de estudiantes y repetir la intervención del estudiante.

Se analiza la asociación entre la intervención del profesor con la intervención de los estudiantes en las clases observadas (Tabla 5), y se encuentra que hay asociación significativa [Chi-Cuadrado $(64, \mathrm{~N}=22524)=27476,903, \mathrm{p}<.001 ; 38$ casillas $(46,9 \%)$ tienen una frecuencia esperada inferior a 5] entre las 9 categorías de intervenciones de los profesores con las 9 categorías de intervenciones de los estudiantes. La fuerza de la dependencia es moderada (V de Cramer=.39).

Se puede afirmar que intervenciones de los profesores e intervenciones de los estudiantes se encuentran en influencia recíproca. Se observa que la mayor cantidad de unidades de tiempo de intervención de los alumnos es para validar conocimientos escolares que entrega el profesor (categoría 1 de estudiantes); más preciso aún, el 82,4\% de esta intervención está asociada a la intervención de presentar conocimientos escolares del profesor (categoría 1 
Estudios Pedagógicos XL, $\mathrm{N}^{\circ}$ 2: 373-389, 2014 DISCURSO Y PRÁCTICA PEDAGÓGICA EN CONTEXTOS DE ALTO RENDIMIENTO ESCOLAR

\begin{tabular}{|c|c|c|c|c|c|c|c|c|c|c|c|c|}
\hline \multirow{10}{*}{ 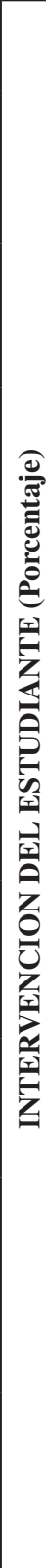 } & 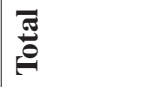 & in & ले & $\stackrel{\infty}{0}$ & 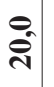 & $\stackrel{b}{i}$ & $\stackrel{10}{\rightarrow}$ & $\stackrel{m}{8}$ & $\stackrel{m}{8}$ & 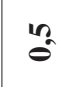 & 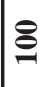 & 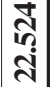 \\
\hline & a & $\frac{0}{\vec{\gamma}}$ & $\vec{\infty}$ & $\overrightarrow{0}$ & $\begin{array}{l}0 \\
0\end{array}$ & $3_{0}^{2}$ & ?. & $\stackrel{0}{0}$ & $\overrightarrow{0}$ & $\overrightarrow{0}$ & \& & 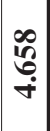 \\
\hline & 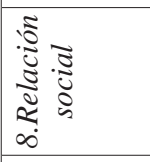 & $\stackrel{m}{i}$ & $\hat{\text { i }}$ & 0 & $\begin{array}{l}0 \\
0\end{array}$ & $\stackrel{\circ}{=}$ & $\stackrel{0}{0}$ & $\stackrel{0}{0}$ & O. & O. & $\stackrel{8}{\Xi}$ & ๙ิ \\
\hline & 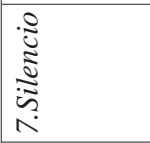 & $\stackrel{\nexists}{ \pm}$ & $\stackrel{n}{\sigma}$ & 0 & $\begin{array}{l}0 \\
0\end{array}$ & in & $\vec{m}$ & $\because$ & $\vec{i}$ & $\stackrel{0}{0}$ & 8 & 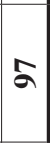 \\
\hline & 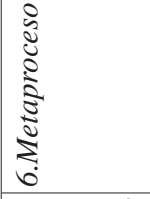 & O. & $\stackrel{\vec{v}}{\tilde{\lambda}}$ & 0 & $\begin{array}{l}0 \\
0\end{array}$ & $\because$ & $\because$ & $\underset{0}{0}$ & O. & 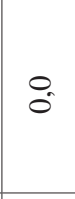 & \& & 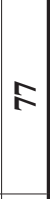 \\
\hline & 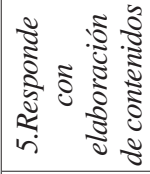 & $\tilde{n}^{n}$ & $\hat{\infty}$ & 0 & $\begin{array}{l}0 \\
0\end{array}$ & $\stackrel{\infty}{0}$ & $\stackrel{0}{-}$ & $\stackrel{0}{0}$ & O. & กু & 8 & 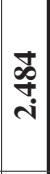 \\
\hline & 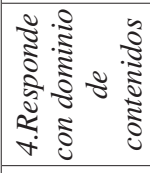 & $\stackrel{\sim}{\underset{J}{J}}$ & $\begin{array}{l}\stackrel{g}{f} \\
\dot{f}\end{array}$ & 0 & $\begin{array}{c}0 \\
i\end{array}$ & ڤ̂̀ & $\hat{i}$ & $\because$ & $\vec{r}$ & $n$ & \& & $\hat{\mathscr{F}}$ \\
\hline & $\begin{array}{l}2 \\
\vdots \\
\vdots \\
2 \\
2 \\
n \\
n\end{array}$ & $\begin{array}{l}\mathscr{n} \\
\dot{J}\end{array}$ & $\hat{i}$ & $\infty$ & $\begin{array}{l}0 \\
0\end{array}$ & $\stackrel{\sim}{-}$ & $\stackrel{0}{\circ}$ & $\stackrel{2}{0}$ & $\dot{\sigma}_{0}^{\circ}$ & $\dot{\sigma}_{0}$ & $\Xi$ & 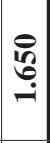 \\
\hline & 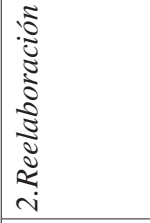 & $\because$ & $\because$ & O. & $O_{0}^{0}$ & $\stackrel{0}{8}$ & $\ddot{0}$ & $\because$ & $\because$ & $\because$ & \& & $\nabla$ \\
\hline & 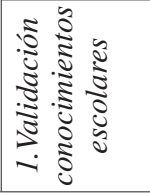 & $\begin{array}{l}\vec{d} \\
\text { di }\end{array}$ & $\begin{array}{l}0 \\
\stackrel{0}{0}\end{array}$ & $\cong$ & $\begin{array}{l}0 \\
\mathrm{i}\end{array}$ & $\hat{i}$ & $\stackrel{i}{i}$ & $\because$ & ô & $\hat{0}$ & ఠ & $\frac{n}{\hat{i}}$ \\
\hline \multicolumn{2}{|c|}{ 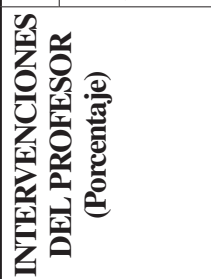 } & 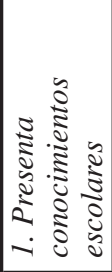 & 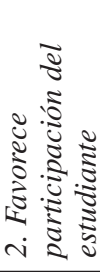 & 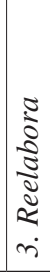 & 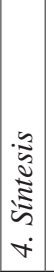 & 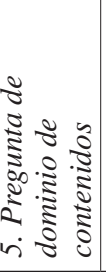 & 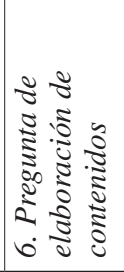 & 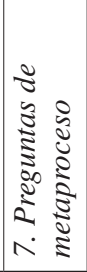 & 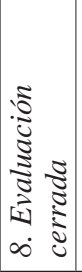 & 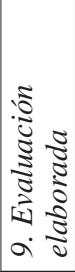 & $\frac{5}{20}$ & हैं \\
\hline
\end{tabular}


del profesor). El cálculo porcentual por casilla indica que el 47,2\% del tiempo que dura la clase se dedica a este tipo de intervenciones. También es interesante notar que la intervención de presentar conocimientos escolares concentra al 72,3\% de intervenciones de la categoría relación social -que equivale a distracción- de los estudiantes, pero esto, en términos del tiempo total de la clase, equivale a menos del $1 \%$.

La intervención de favorecer la participación del estudiante (categoría 2 del profesor) agrupa gran cantidad porcentual de las intervenciones de los estudiantes; el 82\% de las intervenciones de proponer; el $44 \%$ de las intervenciones de responder con dominio de contenidos; el $80 \%$ de las intervenciones de responder con elaboración de contenido, y el $58 \%$ de las intervenciones denominadas en colectivo, que comúnmente refiere al trabajo en grupos de estudiantes en la sala.

El análisis de las casillas indica que en el 12\% del tiempo de las clases observadas, la intervención del profesor para favorecer la participación del estudiante (categoría 2 del profesor) coincide con situaciones donde los estudiantes no establecen una relación dialogal directa con el profesor (categoría 9 de estudiantes). De hecho, éste es el segundo tipo de intervención al cual los estudiantes dedican tiempo, y el 58\% de los mismos están asociados a la categoría 2 del profesor. El tercer grupo de tiempo de las intervenciones de los estudiantes es para responder con elaboración de contenidos (categoría 5 de estudiantes), la cual está asociada a la categoría 2 del profesor: favorecer la participación del estudiante. El cálculo porcentual por casilla indica que el 8,9\% del tiempo total de la clase se produce este encuentro de intervenciones entre profesor y estudiantes.

En síntesis, los resultados presentados indican que cuando el profesor favorece la intervención de los estudiantes éstos diversifican el tipo de intervenciones que realizan en la clase.

\section{CONCLUSIONES}

En los liceos de altos resultados de aprendizaje la cultura escolar tiene normas y procedimientos que organizan la acción de los actores educativos en función de metas de aprendizaje. Dentro de estas condiciones institucionales se puede entender que la intervención del profesor en la clase sea, en más del 50\% del tiempo que dura la clase, de presentar conocimientos escolares. Dicho tiempo es equivalente al tiempo que los alumnos mantienen el interés en la clase, validan, afirman y aceptan los contenidos entregados. Asimismo, cuando el profesor favorece la participación de los estudiantes, éstos diversifican los modos de intervenir en la clase.

La intervención de presentar conocimientos escolares del profesor es hegemónica en las clases observadas, y esto es coherente con las categorías del campo discursivo contextual o de la cultura escolar analizadas en las entrevistas, en lo referente al valor que los profesores dan al orden y sistematicidad con que funciona el liceo. En liceos de alto rendimiento se prioriza la transmisión de contenidos escolares, y la institución se organiza para que eso sea posible. Por ello, el tipo de intervención hegemónica de los estudiantes en la clase está en consonancia con la intervención hegemónica de los profesores. Esto indica que dicha estructura comunicativa es un "saber hacer" que validan ambos interlocutores y tiene como condición la cultura institucional del orden. En dicho escenario, profesores y estudiantes comparten y sostienen recíprocamente las intervenciones que constituyen la interacción profesor-alumnos valorada en el liceo. 
La intervención del profesor del tipo favorece la participación del estudiante también tiene su correlato en la intervención de los estudiantes, que opera como condición y consecuencia para su realización. Dicho tipo de intervención está en consonancia con las subcategorías del campo discursivo de la subjetividad. En efecto, favorecer la participación del estudiante tiene relación con capacidades adquiridas en la formación continua para enseñar la disciplina y con el modelo docente que los profesores describen.

Tal como se ha evidenciado en los estudios sobre creencias de los profesores en torno al aprendizaje, en esta investigación se constata que hay desfase entre lo señalado en el discurso y lo realizado en la práctica real de aula. En efecto, el discurso de los profesores pone el acento en la importancia de conocer y promover la interacción profesor-estudiantes en tanto meta y proceso para lograr el aprendizaje escolar, mientras que en las clases observadas la mayor parte del tiempo es de presentación de conocimientos escolares. No obstante, el discurso del campo de la subjetividad, sobre el modelo docente y las cualidades del profesor en la clase -comunicador efectivo, persona afectuosa y atento a verificar contenidos-, se pone también de manifiesto en el aula a través de la intervención denominada favorece la participación del estudiante, la cual, además, diversifica las formas de participación de los alumnos en la clase.

Futuros estudios deben profundizar en el tipo de intervención del profesor relacionado al conocimiento de la disciplina escolar y la incidencia que esto puede tener en el tipo de intervención de los estudiantes. Del mismo modo, es importante indagar diferenciadamente en las concepciones de los docentes acerca del conocimiento propio de sus áreas disciplinares, a fin de comprender cómo dichas creencias orientarían las planificaciones y prácticas pedagógicas en el desarrollo de distintas asignaturas.

\section{REFERENCIAS BIBLIOGRÁFICAS}

Baeza, J. (2008). El diálogo cultural de la escuela y en la escuela. Estudios Pedagógicos, vol. 34, n. 2, 193-206.

Barría, P., Castro, A., Riveros, F. y Villalta, M. (2010). Experiencia y buenas prácticas pedagógicas en contextos sociales vulnerables de Santiago de Chile: Estudio sobre las creencias de los docentes. Ciencia y Aprendizaje, vol. 2, n. 1, 27-48.

Chan, K. W. \& Elliott, R. (2002). Exploratory Study of Hong Kong Teacher Education Students' Epistemological Beliefs: Cultural Perspectives and Implications on Beliefs Research. Contemporary Educational Psychology, vol. 27, n. 3, 392-414.

MINEDUC. (2010). Sistema Nacional de Evaluación del Desempeño de los Establecimientos Educacionales Subvencionados y de los Regidos por el Decreto Ley $N^{o}$ 3166. Santiago: Ministerio de Educación. Recuperado de http://www.mineduc.cl/usuarios/sned/doc/201108031652460.FolletoDifusionSNED.pdf, consultado en mayo de 2011.

Cobo, P. (1998). Análisis de los procesos cognitivos y de las interacciones sociales entre alumnos (16-17) en la resolución de problemas que comparan áreas de superficies planas. Un estudio de casos. Barcelona: Universitat Autònoma de Barcelona.

Cornejo, R. y Redondo, J. (2007). Variables y factores asociados al aprendizaje escolar. Una discusión desde la investigación actual. Estudios Pedagógicos, vol. 3, n. 2, 155-175.

Cuadrado, I. y Fernández, I. (2008). ¿Cómo intervienen maestros y profesores para favorecer el aprendizaje en secundaria? Un estudio comparativo desde el análisis del discurso. Infancia y Aprendizaje, vol. 31, n. 1, 3-23.

Fernández, I. y Cuadrado, I. (2008). ¿Son conscientes los profesores de secundaria de los 
recursos comunicativos verbales y no-verbales que emplean en el aula? Revista Iberoamericana de Educación, vol. 46, n. 6, 1-13.

García, M. y Sebastián, C. (2011). Creencias epistemológicas de estudiantes de pedagogía en educación parvularia, básica y media: ¿Diferencias en la formación inicial docente? Psykhe, vol. 20, n. 1, 10-23.

Gómez, V. y Guerra, P. (2012). Teorías implícitas respecto a la enseñanza y el aprendizaje: ¿Existen diferencias entre profesores en ejercicio y estudiantes de pedagogía? Estudios Pedagógicos, vol. 38, n. 1, 25-43.

Hargreaves, A. (2005). Profesorado, cultura y postmodernidad: Cambian los tiempos, cambia el profesorado. Madrid: Morata.

Laboratorio Latinoamericano de Evaluación de la Calidad de la Educación (LLECE). (2010). Factores asociados al logro cognitivo de los estudiantes de América Latina y el Caribe. Santiago: UNESCO-OREALC.

Leal, F., Espinoza, C., Iraola, M. y Miranda, M. (2009). El contexto en la epistemología personal: Consideraciones teóricas y exploraciones empíricas. International Journal of Psychology, vol. 43, n. 1, 170-180.

Makuc, M. (2008). Teorías implícitas de los docentes acerca de la comprensión de textos. Signos, vol. 41, n. 68, 403-422.

Marcelo, C. (1989). El pensamiento del profesor. Barcelona: CEAC.

Martin, E., Mateos, M., Martínez, P., Cervi, J., Pecharromán, A. y Villalón, R. (2006). Las concepciones de los profesores de educación primaria sobre la enseñanza y el aprendizaje. En J. Pozo, N. Scheuer, M. Pérez, M. Mateos, E. Martin y M. De la Curz, Nuevas formas de pensar la enseñanza y el aprendizaje. Las concepciones de profesores y alumnos (pp. 171-187). Barcelona: Graò.

Martinic, S. y Vergara, C. (2007). Gestión del tiempo e interacción del profesor-alumno en la sala de clases de establecimientos de Jornada Escolar Completa en Chile. Revista Electrónica Iberoamericana sobre Calidad, Eficacia y Cambio en Educación, vol. 5, n. 5e, 3-20. Recuperado de http://www.rinace.net/arts/vol5num5e/art1.pdf, consultado en junio de 2011.

Medina, J. (2006). La profesión docente y la construcción del conocimiento profesional. Buenos Aires: Editorial Lumen.

Murillo, J. y Román, M. (2009). Mejorar el desempeño de los estudiantes de América Latina. Algunas reflexiones a partir de los resultados del SERCE. Revista Mexicana de Investigación Educativa, vol. 14, n. 41, 451-484.

Nathan, M. \& Kim, S. (2009). Regulation of Teacher Elicitations in the Mathematics Classroom. Cognition and Instruction, vol. 27, n. 2, 91-120.

Navarro, P. y Díaz, C. (1999). Análisis de contenido. En J. Delgado y J. Gutiérrez (Coords.), Métodos y técnicas cualitativas de investigación en ciencias sociales (pp. 177-224). Madrid: Síntesis.

Olafson, L. \& Schraw, G. (2006). Teachers' Beliefs and Practices within and across Domains. International Journal of Educational Research, vol. 45, n. 1-2, 71-84.

Pecharromán, I. y Pozo, J. (2006). ¿Qué es el conocimiento y cómo se adquiere? Epistemología intuitiva en profesores y alumnos de secundaria. En J. Pozo, N. Scheuer, M. Puy, M. Mateos, E. Martin y M. De la Cruz, Nuevas formas de pensar la enseñanza y el aprendizaje. Las concepciones de profesores y alumnos (pp. 243-264). Barcelona: Graò.

Pérez Gómez, Á. (1998). La cultura escolar en la sociedad neoliberal. Madrid: Morata.

Pirela de Faría, L. y Sánchez de Gallardo, M. (2009). Cultura y aprendizaje organizacional en instituciones de Educación Básica. Revista de Ciencias Sociales, vol. 15, n. 1, 175-188.

Programa de las Naciones Unidas para el Desarrollo (PNUD). (2009). Desarrollo humano en Chile. La manera de hacer las cosas. Santiago: PNUD.

Rimmele, R. (2009). What is Videograph? Videograph. The Program for Coding of Videos. Recuperado de http://www.dervideograph.de/enhtmStart.html, consultado en mayo de 2011.

Román, M. (2003). ¿Por qué los docentes no pueden desarrollar procesos de enseñanza 
aprendizaje de calidad en contextos sociales vulnerables? Persona y Sociedad, vol. 17, n. 1, 113-128.

Ruíz, G. y García, A. (2007). Las desigualdades de la escuela y del entorno. Revista Electrónica Iberoamericana sobre Calidad, Eficacia y Cambio en Educación, vol. 5, n. 5e, 306-312.

Strauss, A. y Corbin, J. (2002). Bases de la investigación cualitativa. Técnicas y procedimientos para desarrollar la teoría fundamentada. Colombia: Universidad de Antioquia.

Taylor, S. y Bogdan, R. (1998). Introducción a los métodos cualitativos de investigación. Barcelona: Paidós.

Torrado, J. y Pozo, J. (2006). Del dicho al hecho: De las concepciones sobre el aprendizaje a la práctica de la enseñanza de la música. En J. Pozo, N. Scheuer, M. Pérez, M. Mateos, E. Martin y M. De la Cruz, Nuevas formas de pensar la enseñanza y el aprendizaje. Las concepciones de profesores y alumnos (pp. 205-228). Barcelona: Graò.

UNICEF y Ministerio de Educación de Chile. (2004). ¿Quién dijo que no se puede? Escuelas efectivas en sectores de pobreza. Santiago: Ministerio de Educación-UNICEF. Recuperado de http:// www.unicef.cl/centrodoc/escuelas_efectivas/, consultado en junio de 2011.

Villalta, M. (2014). Cultura escolar y prácticas de enseñanza en establecimientos de alto desempeño educativo. Aceptado en Universitas Psychologica, vol. 13, n. 1. Assael, C. y Martinic, S. (2013). Conocimiento escolar y procesos cognitivos en la interacción didáctica de sala de clase. Aceptado en Perfiles Educativos, vol. 141.

- y Martinic, S. (2011). Interacción didáctica y procesos cognitivos en sala de clase. XXXIII Congreso Interamericano de Psicología, Medellín, Colombia.

., Martinic, S. y Guzmán, M. (2011). Elementos de la interacción didáctica en la sala de clase que contribuyen al aprendizaje en contexto social vulnerable. Revista Mexicana de Investigación Educativa, vol. 16, n. 51, 1137-1158.

(2009). Una propuesta para el estudio de la interacción didáctica en la sala de clase. Estudios Pedagógicos, vol. 35, n. 1, 221-238.

Volante, P., Cumsille, P., Denardin, F. y Müller, M. (2008). Análisis del cambio en los niveles de logro de escuelas de alta vulnerabilidad social. Estudios Pedagógicos, vol. 34, n. 2, 179-191.

Wells, G. \& Mejía, R. (2005). Toward Dialogue in the Classroom: Learning and Teaching through Inquiry. Working Papers on Culture, Education and Human Development, vol. 1, n. 4, 1-45. 
